GLASNIK MATEMATIČKI

Vol. 41(61)(2006), $271-274$

\title{
FINITE NONABELIAN 2-GROUPS IN WHICH ANY TWO NONCOMMUTING ELEMENTS GENERATE A SUBGROUP OF MAXIMAL CLASS
}

\author{
ZVONIMIR JANKO \\ University of Heidelberg, Germany
}

\begin{abstract}
We determine here the structure of the title groups. It turns out that such a group $G$ is either quasidihedral or $\mathrm{G}=\mathrm{HZ}(\mathrm{G})$, where $H$ is of maximal class or extraspecial and $\mho_{1}(Z(G)) \leq Z(H)$. This solves a problem stated by Berkovich. The corresponding problem for $p>2$ is open but very difficult since the $p$-groups of maximal class are not classified for $p>2$.
\end{abstract}

\section{INTRODUCTION AND KNOWN RESULTS}

We determine here the structure of all finite nonabelian 2-groups in which any two noncommuting elements generate a subgroup of maximal class. More precisely, we prove the following result.

THEOREM 1.1. Let $G$ be a finite nonabelian 2-group in which any two noncommuting elements generate a subgroup of maximal class. Then one of the following holds:

(a) $\left|G: H_{2}(G)\right|=2$ and $H_{2}(G)$ is noncyclic (i.e., $G$ is quasidihedral but not dihedral);

(b) $G=H Z(G)$, where $H$ is of maximal class and $\mho_{1}(Z(G)) \leq Z(H)$;

(c) $G=H Z(G)$, where $H$ is extraspecial of order $\geq 2^{5}$ and $\mho_{1}(Z(G)) \leq$ $Z(H)$.

Conversely, each group in (a), (b) and (c) satisfies the assumption of the theorem.

2000 Mathematics Subject Classification. 20D15.

Key words and phrases. Finite 2-groups, 2-groups of maximal class, minimal nonabelian 2-groups, quasidihedral 2-groups, Hughes $H_{p}$-subgroups. 
We consider here only finite $p$-groups and our notation is standard. In particular, a 2-group $S$ is quasidihedral if $S$ has an abelian subgroup $T$ of exponent $>2$ so that $|S: T|=2$ and there is an involution in $S-T$ which inverts each element in $T$. It turns out that $T$ is a characteristic subgroup of $S$.

We state three known results which are used in the proof of Theorem 1.1.

Proposition 1.2 (Berkovich [1, Lemma 4.2]). Let $G$ be a p-group with $\left|G^{\prime}\right|=p$. Then $G=\left(A_{1} * A_{2} * \ldots * A_{s}\right) Z(G)$ (* denotes a central product), where $A_{1}, A_{2}, \ldots, A_{s}$ are minimal nonabelian subgroups.

Proposition 1.3 (Berkovich [1, §58] and Kazarin [2]). Let $G$ be a nonabelian 2-group all of whose cyclic subgroups of composite order are normal in $G$. Then we have either $\left|G: H_{2}(G)\right|=2$ (and then $G$ is quasidihedral) or $\left|G^{\prime}\right|=2$ and the Frattini subgroup $\Phi(G)$ is cyclic.

Proposition 1.4 (Janko [3, Proposition 2.3]). A 2-group is of maximal class if and only if $G$ is dihedral, semidihedral or generalized quaternion.

From Proposition 1.4 follows at once that if $G=\langle a, b\rangle$ is a 2-group of maximal class, then at least one of $a$ and $b$ is of order $\leq 4$ and $G$ possesses exactly one involution $z$ (where $\langle z\rangle=Z(G)$ ) which is a square in $G$. We shall use freely this remark in the proof of Theorem 1.1 .

\section{Proof of Theorem 1.1}

Let $G$ be a nonabelian 2-group in which any two noncommuting elements generate a subgroup of maximal class. We may assume that $G$ is not of maximal class.

(i) First we assume that $\exp (G)>4$. Suppose for a moment that each element of order $\geq 8$ lies in $Z(G)$. Let $x, y \in G$ with $[x, y] \neq 1$. Since $\langle x, y\rangle$ is of maximal class, we have in our case $\langle x, y\rangle \cong D_{8}$ or $\langle x, y\rangle \cong Q_{8}$. Let $k$ be an element of order 8 so that here $k \in Z(G)$. But then $k x$ and $k y$ are elements of order 8 with $[k x, k y]=[x, y] \neq 1$ and therefore $\langle k x, k y\rangle$ is of maximal class, a contradiction. We have proved that $G$ possesses a cyclic subgroup $A$ of order $\geq 8$ such that $A \not Z Z(G)$.

It is easy to see that any cyclic subgroup $X$ of order $\geq 8$ is normal in $G$. Indeed, let $g \in G$ so that $g$ either centralizes $X$ or $\langle X, g\rangle$ is of maximal class in which case $g$ normalizes $X$.

Let $y \in G$ be such that $[A, y] \neq 1$ and so $\langle A, y\rangle$ is of maximal class. Then $\langle A, y\rangle$ contains a subgroup of maximal class $\langle B, y\rangle$ of order $2^{4}$, where $B=\langle b\rangle \cong C_{8}, B \leq A$, and $y^{2} \in \Omega_{1}(B)=\langle z\rangle$. We know that $B$ is normal in $G$. Set $M=C_{G}(B)$ so that $G / M \neq\{1\}$ is elementary abelian of order $\leq 4$. If $G / M \cong E_{4}$, then there is $l \in G-M$ such that $l^{2} \in M, l^{2}$ centralizes $B$ and $b^{l}=b z$. But then $\langle b, l\rangle^{\prime}=\langle z\rangle$ and so $\langle b, l\rangle$ is not of maximal class, a contradiction. Thus $|G: M|=2$. 
For each $x \in G-M, x^{2} \in\langle z\rangle$. Indeed, $[b, x] \neq 1$ and so $\langle b, x\rangle$ is of maximal class and therefore $x^{2} \in \Omega_{1}(B)=\langle z\rangle$. Consider $\bar{G}=G /\langle z\rangle$. Then all elements in $\bar{G}-\bar{M}$ are involutions which implies that $M /\langle z\rangle$ is abelian and for each $m \in M, m^{y}=m^{-1} z^{\epsilon}, \epsilon=0,1$.

Suppose that $M$ is nonabelian. Then $M^{\prime}=\langle z\rangle$ and let $m, n \in M$ with $[m, n]=z$. In that case (since $\langle m, n\rangle$ is of maximal class), $\langle m, n\rangle \cong D_{8}$ or $\cong Q_{8}$. But then $b m$ and $b n$ are elements of order 8 with $[b m, b n]=[m, n]=z$ and so $\langle b m, b n\rangle$ is of maximal class, a contradiction. Hence $M$ is abelian. If $M$ is cyclic, then $\langle M, y\rangle=G$ is of maximal class, a contradiction. Thus, $M$ is noncyclic abelian.

If all elements in $G-M$ are involutions, then $H_{2}(G)=M$ and we have obtained a group in part (a) of our theorem.

We may assume that not all elements in $G-M$ are involutions and so we may suppose $y^{2}=z$. Let $t$ be any involution in $M-\langle z\rangle$ and assume that $t$ is a square in $M$, i.e., there is $k \in M$ such that $k^{2}=t$. Since $k^{y}=$ $k^{-1} z^{\epsilon}(\epsilon=0,1),\langle y, k\rangle$ is nonabelian. In that case $\langle y, k\rangle$ is of maximal class containing two distinct involutions $z$ and $t$ which are squares in $\langle y, k\rangle$, a contradiction. We have proved that $M$ is abelian of type $\left(2^{s}, 2, \ldots, 2\right), s \geq 3$. Setting $E=\Omega_{1}(M)$, we get $M=\left\langle b^{\prime}\right\rangle E$, where $o\left(b^{\prime}\right)=2^{s},|E| \geq 4$, and $\left\langle b^{\prime}\right\rangle \cap E=\Omega_{1}\left(\left\langle b^{\prime}\right\rangle\right)=\langle z\rangle$ since $z$ is the unique involution in $M$ which is a square in $M$. Since $\left(b^{\prime}\right)^{y}=\left(b^{\prime}\right)^{-1} z^{\eta}(\eta=0,1), H=\left\langle b^{\prime}, y\right\rangle$ is of maximal class and $G=H E$. For each $t \in E$, we have either $t^{y}=t$ or $t^{y}=t z$. If $y$ centralizes $E$, then $E=Z(G)$. If $y$ does not centralize $E$, then $E_{0}=C_{E}(y)$ is of index 2 in $E$. Let $v$ be an element of order 4 in $\left\langle b^{\prime}\right\rangle$ and let $u \in E-E_{0}$. In that case

$$
(v u)^{y}=v^{-1}(u z)=(v z)(u z)=v u \text { and }(v u)^{2}=z,
$$

and so $Z(G)=E_{0}\langle v u\rangle$ with $\mho_{1}(Z(G))=\langle z\rangle$. In any case we get $G=H Z(G)$, $Z(G)>Z(H)=\langle z\rangle$, and $\mho_{1}(Z(G)) \leq\langle z\rangle$. We have obtained a group in part (b) of our theorem.

(ii) We examine now the case $\exp (G)=4$. Let $\langle x\rangle$ be a cyclic subgroup of order 4 and $y \in G$. Then either $[x, y]=1$ or $\langle x, y\rangle \cong D_{8}$ or $Q_{8}$. In any case $y$ normalizes $\langle x\rangle$ and so each cyclic subgroup of order 4 is normal in $G$. We may use Proposition 1.3. It follows that either $\left|G: H_{2}(G)\right|=2$ (and we get a group of part (a) of our theorem) or $\left|G^{\prime}\right|=2$ and $\Phi(G)$ is cyclic.

Suppose that we are in the second case. Since $G$ does not possess elements of order 8, we have $|\Phi(G)|=2$ and then $\Phi(G)=G^{\prime}$. The fact $\left|G^{\prime}\right|=2$ implies that $G=H_{1} * H_{2} * \ldots * H_{n} Z(G)$, where $H_{i}(i=1, \ldots, n)$ is minimal nonabelian (Proposition 1.2). In our case $H_{i} \cong D_{8}$ or $Q_{8}$ and so $H=H_{1} * H_{2} * \ldots * H_{n}$ is extraspecial. Also, $\Phi(G)=G^{\prime}=H^{\prime}=Z(H)$ implies that $\mho_{1}(Z(G)) \leq Z(H)$. If $n=1$, we have obtained a group of part (b) of our theorem and so we may assume that $n>1$. In that case $|H| \geq 2^{5}$ and we have obtained a group of part (c) of our theorem. 
It is necessary to prove only for groups (b) and (c) of our theorem that any two noncommuting elements generate a group of maximal class. Indeed, let $h_{1} z_{1}$ and $h_{2} z_{2}$ be any noncommuting elements in $G$, where $h_{1}, h_{2} \in H$ and $z_{1}, z_{2} \in Z(G)$. Then $\left[h_{1} z_{1}, h_{2} z_{2}\right]=\left[h_{1}, h_{2}\right] \neq 1$ and so $H_{0}=\left\langle h_{1}, h_{2}\right\rangle \leq H$ is a group of maximal class with $H_{0}^{\prime} \geq Z(H)$. On the other hand, a 2-group $\left\langle h_{1}, h_{2}\right\rangle$ is of maximal class if and only if $\left[h_{1}, h_{2}\right] \neq 1,\left\langle\left[h_{1}, h_{2}\right]\right\rangle$ is normal in $H_{0}$ and $h_{1}^{2}, h_{2}^{2} \in\left\langle\left[h_{1}, h_{2}\right]\right\rangle$. Hence $H_{1}=\left\langle h_{1} z_{1}, h_{2} z_{2}\right\rangle$ is of maximal class since $\left[h_{1} z_{1}, h_{2} z_{2}\right]=\left[h_{1}, h_{2}\right] \neq 1, h_{1} z_{1}$ and $h_{2} z_{2}$ normalize $\left\langle\left[h_{1} z_{1}, h_{2} z_{2}\right]\right\rangle=\left\langle\left[h_{1}, h_{2}\right]\right\rangle$ and $\left(h_{1} z_{1}\right)^{2},\left(h_{2} z_{2}\right)^{2}$ are contained in $\left\langle\left[h_{1}, h_{2}\right]\right\rangle$ (noting that $z_{1}^{2}, z_{2}^{2} \in Z(H) \leq$ $\left.H_{0}^{\prime}=H_{1}^{\prime}\right)$.

\section{REFERENCES}

[1] Y. Berkovich, Groups of prime power order, Parts I, II, and III (with Z. Janko) in preparation.

[2] L. S. Kazarin, Groups with certain conditions imposed on the normalizers of subgroups, Perm. Gos. Univ. Učen. Zap. 218 (1969), 268-279 (Russian).

[3] Z. Janko, Finite 2-groups with small centralizer of an involution, J. Algebra 241 (2001), 818-826.

Z. Janko

Mathematical Institute

University of Heidelberg

69120 Heidelberg

Germany

E-mail: janko@mathi.uni-heidelberg.de

Received: 5.4.2006. 